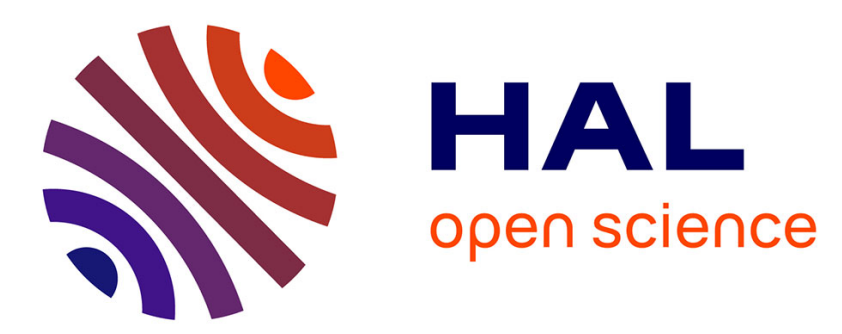

\title{
Small Scale Statistics of Turbulent Fluctuations Close to a Stagnation Point
}

\author{
Peter Dearborn Huck, Nathanaël Machicoane, Romain Volk
}

\section{To cite this version:}

Peter Dearborn Huck, Nathanaël Machicoane, Romain Volk. Small Scale Statistics of Turbulent Fluctuations Close to a Stagnation Point. Turbulent Cascades II, 2019, 10.1007/978-3-030-125479_14. hal-02515777

\author{
HAL Id: hal-02515777 \\ https://hal.science/hal-02515777
}

Submitted on 26 Mar 2020

HAL is a multi-disciplinary open access archive for the deposit and dissemination of scientific research documents, whether they are published or not. The documents may come from teaching and research institutions in France or abroad, or from public or private research centers.
L'archive ouverte pluridisciplinaire HAL, est destinée au dépôt et à la diffusion de documents scientifiques de niveau recherche, publiés ou non, émanant des établissements d'enseignement et de recherche français ou étrangers, des laboratoires publics ou privés. 


\title{
Small Scale Statistics of Turbulent Fluctuations Close to a Stagnation Point
}

Peter D. Huck, Nathanael Machicoane and Romain Volk

\begin{abstract}
Experimental data measured with a 3d Shadow-Particle Tracking Velocimetry (S-PTV) setup in fully developed turbulence $\left(\operatorname{Re}_{\lambda}=[175-225]\right)$ is presented. The underlying flow is of the von Kármán type and as other similar flows, its mean flow is bistable, the two states having the topology of a stagnation point with one contracting and two dilating directions. Tracer particle trajectories permit the investigation of the inhomogeneity and anisotropy of the smallest scales, namely acceleration statistics. The local variance and time-scale of acceleration components are shown to mimic the large scale properties of the flow, the time-scales being more anisotropic than the variances. We explain the hierarchy of time-scales by investigating the Lagrangian Taylor micro-scale which is related to acceleration and velocity variances, and discuss the very high Reynolds number regime.
\end{abstract}

\section{Introduction}

Inhomogeneity in fluid flows is inherent in natural and industrial contexts with examples in the free shear and convection of the planetary boundary layer [1] or rotation in stirred chemical reactors [2] and compression in piston engines [3]. Among the canonical flow types investigated in the literature, strain is an important mechanism that has been used to investigate the link between the imposed mean-field and the resulting anisotropy $[4,5]$ and theoretical formulation of the role of rapidly applied strain to turbulence succeeded in providing a mechanism to predict anisotropy [6].

Recent experimental [7] and numerical [8] investigations have demonstrated that

P. D. Huck · R. Volk $(\varangle)$

Laboratoire de Physique de l'Ecole Normale Supérieure de Lyon, CNRS,

Université de Lyon, Lyon, France

e-mail: romain.volk@ens-lyon.fr

P. D. Huck

e-mail: peter.huck@ens-lyon.fr

N. Machicoane

Department of Mechanical Engineering, University of Washington, Seattle, USA

e-mail:nmachico@uw.edu

M. Gorokhovski and F. S. Godeferd (eds.), Turbulent Cascades II,

ERCOFTAC Series 26, https://doi.org/10.1007/978-3-030-12547-9_14 
accurate prediction is limited to larger scales when applied deformation is not sufficiently rapid to attain the small dissipative regions of the turbulence. Only recently have simulations investigated the implications of strain on the small dissipative scales of turbulence [9] demonstrating stronger root-mean-square ( $\mathrm{rms}$ ) acceleration in compressed directions than in divergent directions in asymmetric strain. Interestingly, recent study has not lead to conclusive explanations of the role such flow conditions have on dissipative scale temporal dynamics.

In this contribution we build upon these concepts with an experimental Lagrangian investigation of fully developed turbulence $\left(\operatorname{Re}_{\lambda}=[175-225]\right)$. In particular, a highly inhomogeneous and anisotropic von Kármán type flow is studied. Though often thought to belong to the free-shear category [10], recent study has pointed to the fundamental role of the stagnation point at the center of the flow [11]. By way of analogy with the characterization of spatial velocity gradients (Eulerian Taylor scale, $\lambda$ ) [12], the Lagrangian Taylor scale [13] is used to characterize high frequency motions and provides a reasonably accurate estimation of acceleration anisotropy, both in terms of temporal correlation and amplitude.

\section{Experimental Set-Up}

The device used in this investigation is the so-called von Kármán flow which consists of a square cylindrical enclosure, $15 \mathrm{~cm}$ on each side, with two counter-rotating disks of radius $R=7.1 \mathrm{~cm}$ driven at equal rotation frequencies by constant-current motors that are separated by $20 \mathrm{~cm}$, as depicted in Fig. 1a. Our experiments rely on a ShadowParticle Tracking Velocimetry [14] where two perpendicular collimated beams permit the tracking of small objects over a large volume [11] approximately $(6 \mathrm{~cm})^{3}$ (Fig. 1b). Trajectories are reconstructed using typical particle tracking algorithms [15] applied to films obtained with two high speed cameras (Phantom V.12, Vision Research, 1Mpix@7 kHz) with a resolution $800 \times 768$ pixels, and a frame rate of $f_{s}=12 \mathrm{kHz}$.

A vast literature exists on the bistable nature of the von Kármán flow (e.g. [16, 17]) measured primarily in round cylinder geometries. The present square cylinder manifests another type of bistability for which the lifetime of the two states (characterized from LDV measurements, not shown here) is much longer than the duration of each movie. A explained in [14], it is possible to separate S-PTV data into two ensembles, each one corresponding to one of the two states, using the kinetic energy contained in each velocity component averaged over the ensemble of trajectories measured in a single film. Such conditioning allows for a reconstruction of averaged flow properties in $3 \mathrm{D}$ for each states such as the mean flow as shown in Fig. $1 \mathrm{~b}$ for the case of the $\mathrm{x}$-dominant state. The topology of each state have a peculiar topology (see [14] for more details): the $\mathrm{x}$-dominant state, which will be investigated in the following, presents a stagnation point near the origin with one stable direction $(x)$ and two unstable directions ( $y$ and $z$ ) as depicted in Fig. 1b, c, while $x$ and $y$ directions are exchanged in the y-dominant state (Fig. 1d). We note that the volume over which 
(a)

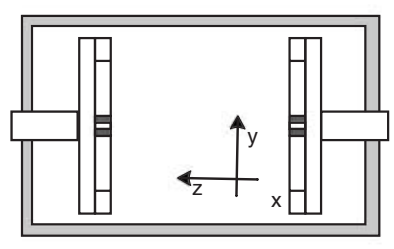

(b)

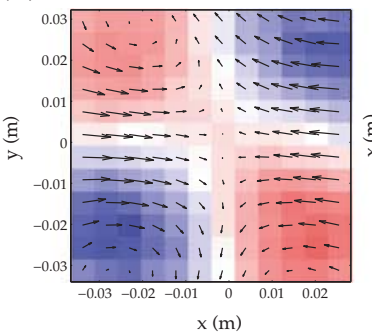

x-dominant state



(c)

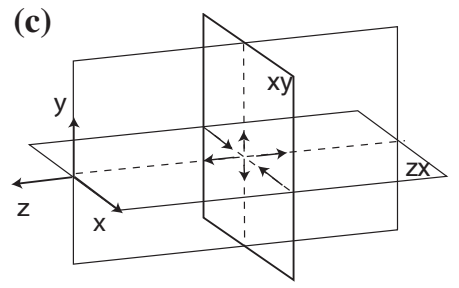

(d)

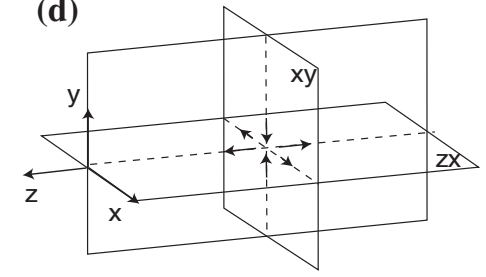

Fig. 1 Experimental apparatus. a The square cylindrical enclosure of the von Kármán flow consists of two counter rotating disks driven at equal rotational frequencies. $\mathbf{b}$ Reconstructed mean flow for the $\mathrm{x}$-dominant state. Left: arrows indicate $\left(\left\langle\mathrm{v}_{x}\right\rangle,\left\langle\mathrm{v}_{y}\right\rangle\right)$ measured in the $(x y)$ plane, the color coding for $\left\langle\mathrm{v}_{z}\right\rangle$. Right: arrows indicate $\left(\left\langle\mathrm{v}_{z}\right\rangle,\left\langle\mathrm{v}_{x}\right\rangle\right)$ measured in the $(z x)$ plane, the color coding for $\left\langle\mathrm{v}_{y}\right\rangle$. c Sketch of the mean flow orientation in the x-dominant state. $\mathbf{d}$ Sketch of the mean flow orientation in the y-dominant state.

Table 1 Parameters of the flow. $\Omega$, rotation rate of the discs; $\varepsilon$, dissipation rate obtained from the power consumption of the motors. The kinematic viscosity of the water-Ucon ${ }^{\mathrm{TM}}$ mixture is $v=8.2 \times 10^{-6} \mathrm{~m}^{2} \mathrm{~s}^{-1}$ with a density $\rho=1000 \mathrm{~kg} \mathrm{~m}^{-3}$. The dissipative time-scale and lengthscale are $\tau_{\eta}=\sqrt{v / \varepsilon}$ and $\eta=\left(v^{3} / \varepsilon\right)^{1 / 4}$, the Taylor-based Reynolds number being estimated as $\operatorname{Re}_{\lambda}=\sqrt{15 \mathrm{v}^{\prime 4} / \nu \varepsilon}$ with $\mathrm{v}^{\prime}=\sqrt{\left(\left\langle\mathrm{v}^{\prime}{ }^{2}\right\rangle+\left\langle\mathrm{v}^{\prime}{ }^{2}\right\rangle+\left\langle\mathrm{v}_{z}^{\prime}{ }^{2}\right\rangle\right) / 3}$ and $R e=2 \pi R^{2} \Omega / v$

\begin{tabular}{l|l|l|l|l|l|l}
\hline$\Omega(\mathrm{Hz})$ & $\mathrm{v}^{\prime}\left(\mathrm{ms}^{-1}\right)$ & $\tau_{\eta}(\mathrm{ms})$ & $\eta(\mu \mathrm{m})$ & $\begin{array}{l}\varepsilon_{m} \\
\left(\mathrm{~W} \mathrm{~kg}^{-1}\right)\end{array}$ & $R e_{\lambda}(-)$ & $\operatorname{Re}(-)$ \\
\hline 4.2 & 0.34 & 3.2 & 162 & 0.8 & 175 & 16,200 \\
\hline 5.5 & 0.45 & 2.1 & 131 & 1.9 & 200 & 21,200 \\
\hline 6.9 & 0.56 & 1.5 & 111 & 3.6 & 225 & 26,700 \\
\hline
\end{tabular}

the statistics are computed is larger than the Eulerian integral scale $L=\mathrm{v}^{\prime 3} / \varepsilon=4.8$ $\mathrm{cm}$, where $\mathrm{v}^{\prime}$ is the rms value of the fluctuating velocity, permitting an investigation of their inhomogeneity. Various Eulerian statistics are given at the geometrical center of the flow in Table 1. 


\subsection{Inhomogeneity in the Acceleration Magnitude}

The stagnation point is responsible for a large amplification of fluctuations in the contracting direction $(x)$ while attenuation is observed in the dilating directions [11]. As a consequence the total velocity fluctuations $\left\langle\mathrm{v}^{\prime 2}\right\rangle$ increase as particles approach the stagnation point (Fig. 2a), indicating spatial inhomogeneity, with an anisotropy level $\left\langle\mathrm{v}^{\prime 2}{ }_{x}\right\rangle /\left\langle\mathrm{v}^{\prime 2}{ }_{z}\right\rangle$ which nearly doubles as the stagnation point is reached (Fig. 2a, inset).

As displayed in Fig. 2b, the acceleration component magnitude (normalized by its maximum value at the center to account its Reynolds number dependence) is also found to increase when approaching the center. Such spatial profile of acceleration fluctuations is reminiscent of the non homogeneity in average dissipation, which was found maximum near the geometrical center [11]. This is because acceleration variance is expected to depend on dissipation following the Heisenberg-Yaglom relation $\left\langle a_{i}^{2}\right\rangle=a_{0, i} \varepsilon^{3 / 2} v^{-1 / 2}$ [18], where $a_{0, i}$ is a non-dimensional function of the Reynolds number. As opposed to the increasing anisotropy of velocity fluctuations, the three components of the fluctuating acceleration increase in equal proportions so that anisotropy is constant over the entire region of Fig. 2b with the hierarchy $\left\langle a_{x}{ }^{2}\right\rangle>\left\langle a_{y}{ }^{2}\right\rangle>\left\langle a_{z}{ }^{2}\right\rangle$. This shows that the anisotropy observed in the large scale quantities propagates down to the very small scales of turbulent fluctuations as was observed in [15].

(a)

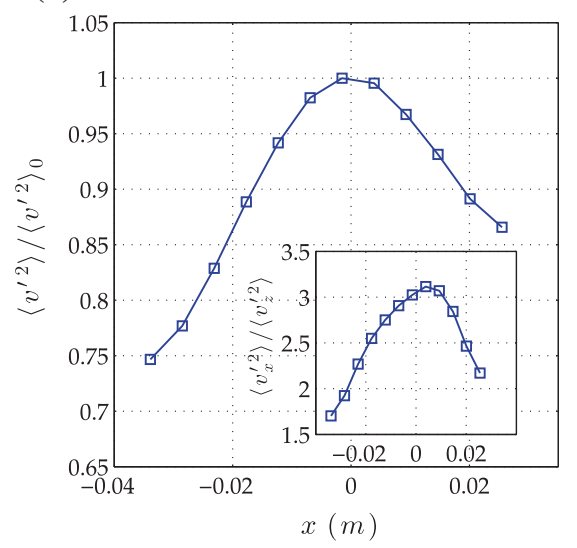

(b)

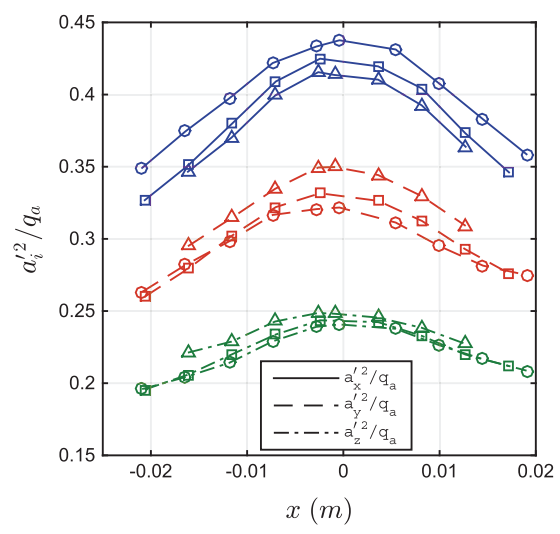

Fig. 2 Velocity fluctuations statistics at $R e_{\lambda}=200$. a Overall increase in velocity fluctuations $\left(\left\langle\mathrm{v}^{\prime 2}\right\rangle=\left(\left\langle\mathrm{v}_{x}^{\prime}{ }^{2}\right\rangle+\left\langle\mathrm{v}_{y}^{\prime}{ }^{2}\right\rangle+\left\langle\mathrm{v}_{z}^{\prime}{ }^{2}\right\rangle\right) / 3\right)$ normalized by its value at the origin. Inset: anisotropy between the converging $\left(\left\langle\mathrm{v}_{x}^{\prime 2}\right\rangle\right)$ and diverging $\left(\left\langle\mathrm{v}_{z}^{\prime 2}\right\rangle\right)$ fluctuations. b Acceleration magnitude statistics. $\circ: R e_{\lambda}=175, \square: R e_{\lambda}=200, \triangleleft: R e_{\lambda}=225$. a Normalized acceleration variance where $q_{a}=\left(\left\langle a_{x}{ }^{2}\right\rangle+\left\langle a_{y}{ }^{2}\right\rangle+\left\langle a_{z}{ }^{2}\right\rangle\right)$ is evaluated at $(0,0,0)$. 


\subsection{Acceleration Time Scales and Anisotropy}

We now investigate the temporal dynamics at small scale and focus on the autocorrelation function of the acceleration components $R_{a}^{i}(\tau)=\left\langle a_{i}(t) a_{i}(t+\tau)\right\rangle /\left\langle a^{2}\right\rangle$. Because the flow is non homogeneous, we restrict to trajectories passing through a ball of $1 \mathrm{~cm}$ in diameter whose center is located at the origin $(0,0,0)$. Figure 3 a plots the auto-correlation function versus normalized time $\tau / \tau_{\eta}$ where $\tau_{\eta}=(v / \varepsilon)^{1 / 2}$ takes the Reynolds number dependence into account and permits a collapse of the three curves. The plots show that the temporal dynamics is anisotropic since the curves do not have the same zero crossing time $t_{0}$. In order to get a robust estimate of the acceleration characteristic time, we measure the integral time $\tau_{a, i}=\int_{0}^{t_{0}} R_{a}^{i}(\tau) \mathrm{d} \tau$ for each component $i=x, y, z$.

The inset of Fig. 3a displays the normalized acceleration integral time obtained for different locations of the ball center along the $x$ axis. As stagnation point is approached, the integral time $\tau_{a, i}$ decreases. This is consistent with an increase of dissipation close to the geometrical center as this time scale is expected to be proportional to $\tau_{\eta}=\sqrt{v / \varepsilon}$ as shown in homogeneous and isotropic turbulence (HIT) [19]. However, we observe $\tau_{a, i}$ become increasingly anisotropic and attain a maximum at the flow geometric center with the hierarchy $\tau_{a, x} / \tau_{a, z}>1$ whereas $\left\langle a_{x}{ }^{2}\right\rangle>\left\langle a_{z}{ }^{2}\right\rangle$. This observation is somewhat counter-intuitive when considering $\tau_{a} \simeq \sqrt{\nu / \varepsilon}$ which would suggest a scaling law $\tau_{a} \propto\left(\nu /\left\langle a^{2}\right\rangle\right)^{1 / 3}$. However, the opposite is observed;

(a)



(b)

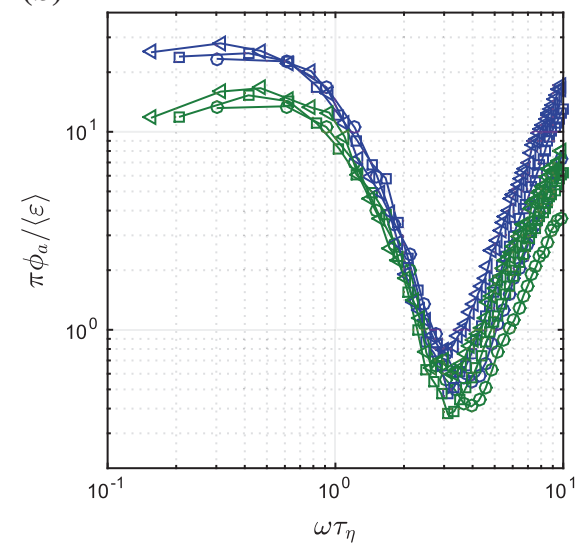

Fig. 3 Acceleration auto-correlation and spectra at the stagnation point. $\circ: R e_{\lambda}=175, \square: R e_{\lambda}=$ $200, \triangleleft: R e_{\lambda}=225$. a Acceleration correlation function ( $a_{x}$, blue, $a_{z}$ green) near the stagnation point for all Reynolds numbers. Inset: acceleration integral time normalized by the dissipative time-scale $\tau_{\eta}=\sqrt{\nu / \varepsilon}$. The black curves are averages of the three Reynolds numbers to serve as a reference. $\mathbf{b}$ Unfiltered acceleration spectra at the stagnation point normalized using the dissipation ( $a_{x}$, blue, $a_{z}$ green). 
the strongest component $(x)$ has the largest acceleration time whereas the weakest component $(z)$ has the smallest time-scale.

In order to understand how acceleration variance and time-scale are influenced by the large scales, we display in Fig. $3 \mathrm{~b}$ the acceleration spectra of $a_{x}$ and $a_{z}$, computed directly from the raw acceleration trajectories. The spectra are related to the autocorrelation functions by the following relationship:

$$
\phi_{a, i}(\omega)=\frac{2}{\pi} \int_{0}^{\infty} R_{a}^{i}(\tau) \cos (\omega \tau) \mathrm{d} \tau .
$$

They have been normalized by $\varepsilon \pi^{-1}$ which has been used in the literature to account for $R e_{\lambda}$ dependence [20,21]. This figure shows that anisotropy is contained in the low frequencies below $\omega \tau_{\eta} \simeq 1$ while higher frequencies in the deep dissipative region $\left(\omega \tau_{\eta}>1\right)$ become isotropic. It is seen from this figure that integral quantities such as acceleration variance $\left\langle a_{i}{ }^{2}\right\rangle=\int_{0}^{\infty} \phi_{a, i}(\omega) \mathrm{d} \omega$ and acceleration time-scale have a contribution from low frequencies, i.e. from scales larger than the dissipative scales, which are non isotropic.

It is not evident from the acceleration spectrum to estimate the acceleration time-scale, but its hierarchy may be understood by computing the Lagrangian Taylor scale $\tau_{L}$ (not to be confused with the eulerian Taylor microscale $\lambda$ ), which is defined from the short time evolution of the velocity auto-correlation function $1 / \tau_{L}^{2}=-1 / 2 \mathrm{~d} R_{L} / d \tau^{2}(0)$. Indeed, this time scale can be linked to the acceleration and velocity variances through the relationship

$$
\left\langle a_{i}{ }^{2}\right\rangle R_{a}^{i}(\tau)=-\left\langle\mathrm{v}_{i}^{\prime 2}\right\rangle \frac{\mathrm{d}^{2}}{\mathrm{~d} \tau^{2}} R_{L}^{i}(\tau),
$$

which is valid for statistically stationary signals. The Lagrangian Taylor time-scale of component $i$ then writes $\tau_{L, i}^{2}=2\left\langle\mathrm{v}_{i}^{\prime 2}\right\rangle /\left\langle a_{i}{ }^{2}\right\rangle$, which shows that this time-scale is influenced both by large and small scales. Although $\tau_{L}$ is not the same as $\tau_{a}$, the latter is only slightly larger than the former, i.e. in the central region $\tau_{a i} \simeq[1.25-1.45] \tau_{\eta}$ and $\tau_{L, i} \simeq[4-6] \tau_{\eta}$ depending on the component observed. As such, $\tau_{L}$ falls squarely in the region of frequencies most strongly contributing to the anisotropy as seen in the spectra of Fig. 3b. Anisotropy can thus be estimated: $\tau_{a, x} / \tau_{a, z} \simeq 1.13$ as measured by the integral time scales in Fig. 3(a inset) while $\tau_{L, x} / \tau_{L, z} \simeq 1.33$ as estimated from $\tau_{L, i}^{2}=2\left\langle\mathrm{v}_{i}^{\prime 2}\right\rangle /\left\langle a_{i}{ }^{2}\right\rangle$. The Taylor timescale over predicts the anisotropy by roughly $20 \%$ and explains why acceleration time-scales are less isotropic than acceleration variances.

In a similar flow velocity anisotropy ratio has been shown to decay slowly to just below $\mathrm{v}^{\prime}{ }_{x} / \mathrm{v}^{\prime}{ }_{z} \simeq 1.5$ at $R e_{\lambda} \simeq 1000$ [22] while the ratio of acceleration rms decays to nearly 1.1 . However, as shown above, time scales imply interaction of both large (velocity) and small (acceleration) scales. Consequently, the persistent anisotropy in the large scales resulting from the presence of the stagnation point inhibit isotropization of time scales at large Reynolds numbers. Indeed, Lagrangian 
measurements spanning $R e_{\lambda}=[450-810]$ indicate very little evolution in $\tau_{a x} / \tau_{a z}$ [23].

\section{Conclusion}

This article presented an experimental investigation of Lagrangian data in the fully developed turbulence of a von Kármán flow. The flow presents a bistability and each state can be investigated separately by conditioning on the kinetic energy contained in each component. Restricting the data analysis to only one state, the turbulent velocity fluctuations were seen to be strongly inhomogeneous close to the stagnation point. Additionally, anisotropy measured between the converging and weakest diverging direction increased monotonically. The turbulent dissipation rate was observed to be dominated by the converging direction and mirrored the evolution of the turbulent velocity fluctuations. Interestingly, the acceleration variance followed the tendency of the turbulent dissipation and velocity fluctuations to increase, though unlike the latter, acceleration anisotropy remained almost constant.

Although the relationship small scale anisotropy can be linked to the large scales, this relationship is incapable of predicting anisotropy among the acceleration timescales. The hierarchy of time-scales was explained by investigating the Lagrangian Taylor scale $\tau_{L}=\sqrt{2 \mathrm{v}^{\prime 2} / a^{2}}$. The presence of $\mathrm{v}^{\prime}$ in this relationship suggests an influence from lower frequencies, which is confirmed from the inspection of acceleration spectra. Frequencies close to $\omega=2 \pi / \tau_{L}$ contribute most strongly to anisotropy in the acceleration magnitude whereas the deep dissipative region $\left(\omega \tau_{\eta}>1\right)$ becomes isotropic. Similar behavior is apparent in the acceleration auto-correlation which by consequence lead to anisotropic measurements of its integral scale $\tau_{a}$.

The literature contains sparse discussion of the anisotropy of small scale statistics and this study proposes a framework in which they may be understood. The derivation of $\tau_{L}$ follows from the kinematic relationship between velocity and acceleration auto-correlation functions and is expected to not only hold for the fluid particle tracers studied here, but for particles whose dynamics are dominated by their inertia. Further study into the effects of inhomogeneity and anisotropy for these particle classes is of great interest for the atmospheric dispersion of pollutants $[18,24]$ and the process of rain and ice formation.

Acknowledgements This work is supported by research programs ANR-13-BS09-0009 and PALSE/2013/26. Contribution from the EuHIT-European High-performance Infrastructures in Turbulence-is also acknowledged. 


\section{References}

1. Sawford, B.L.: Lagrangian statistical simulation of concentration mean and fluctuation fields. J. Clim. Appl Meteorol. 24, 1152-1166 (1985)

2. Paul, E.L., Atiemo-Obeng, V.A., Kresta, S.M.: Handbook of Industrial Mixing: Science and Practice. Wiley , Inc. (2004)

3. Breuer, S., Oberlack, M., Peters, N.: Non-isotropic length scales during the compression stroke of a motored piston engine. Flow Turbul. Combust. 74(2), 145-167 (2005)

4. Comte-Bellot, G., Corrsin, S.: The use of a contraction to improve the isotropy of grid-generated turbulence. J. Fluid Mech. 25(04), 657 (1966)

5. Tucker, H.J., Reynolds, A.J.: The distortion of turbulence by irrotational plane strain. J. Fluid Mech. 32, 657 (1968)

6. Sagaut, P., Cambon, C.: Homogeneous Turbulence Dynamics. Cambridge University Press (2008)

7. Ayyalasomayajula, S., Warhaft, Z.: Nonlinear interactions in strained axisymmetric highReynolds-number turbulence. 566(2006), 273 (2006)

8. Clay, M.P., Yeung, P.K.: A numerical study of turbulence under temporally evolving axisymmetric contraction and subsequent relaxation. J. Fluid Mech. 805, 460-493 (2016)

9. Lee, C.-M., Gylfason, Á., Perlekar, P., Toschi, F.: Inertial particle acceleration in strained turbulence. J. Fluid Mech. 785, 31-53 (2015)

10. Marie, L., Daviaud, L.: Experimental measurement of the scale-by-scale momentum transport budget in a turbulent shear flow. Phys. Fluids 16(2) (2004)

11. Huck, P.D., Machicoane, N., Volk, R.: Production and dissipation of turbulent fluctuations close to a stagnation point. Phys. Rev. Fluids 2(8), 084601 (2017)

12. Taylor, G.I.: Statistical theory of turbulence. Proc. Roy. Soc. A 151(873), 421-478 (1935)

13. Corrsin, S., Uberoi, M.: Diffusion of heat from a line source in isotropic turbulence. Natl. Adv. Comm. Aeronaut. Tech. Rep. 1142(1142) (1953)

14. Huck, P.D., Machicoane, N., Volk, R.: A cost-efficient shadow particle tracking velocimetry setup suitable for tracking small objects in a large volume. Procedia IUTAM 20, 175-182 (2017)

15. Ouellette, N.T., Xu, H., Bodenschatz, E.: A quantitative study of three-dimensional Lagrangian particle tracking algorithms. Exper. Fluids 40(2), 301-313 (2006)

16. De La Torre, A., Burguete, J.: Slow dynamics in a turbulent von Kármán swirling flow. Phys. Rev. Lett. 99(5), 3-6 (2007)

17. Ravelet, F., Chiffaudel, A., Daviaud, F.: Supercritical transition to turbulence in an inertially driven von Kármán closed flow. J. Fluid Mech. 601, 339-364 (2008)

18. Sawford, B.L., Pinton, J.F.: A Lagrangian view of turbulent dispersion and mixing. In: Sreenviasan, K.R., Davidson, P.A., Kaneda, Y. (eds.) Ten Chapters in Turbulence, Chapter 4, pp. 132-175. Cambridge University Press, Cambridge (2012)

19. Yeung, P.K., Pope, S.B.: Lagrangian statistics from direct numerical simulations of isotropic turbulence. J. Fluid Mech. 207, 531 (1989)

20. Sawford, B.L., Yeung, P.K.: Kolmogorov similarity scaling for one-particle Lagrangian statistics. Phys. Fluids 23(9), 1-5 (2011)

21. Ouellette, N.T., Xu, H., Bourgoin, M., Bodenschatz, E.: Small-scale anisotropy in Lagrangian turbulence. New J. Phys. 8 (2006)

22. Voth Greg, A., La Porta, A., Crawford, A.M., Alexander, J., Bodenschatz, E.: Measurement of particle accelerations in fully developed turbulence. J. Fluid Mech. 469, 121-160 (2002)

23. Volk, R., Chareyron, D., Pinton, J.: Mesures d'accélération lagrangienne dans un écoulement anisotrope par vélocimétrie laser Doppler étendue. 20ème Congrès Français De Mécanique, pp. 1-6 (2011)

24. Toschi, F., Bodenschatz, E.: Lagrangian properties of particles in turbulence. Ann. Rev. Fluid Mech. 41, 375-404 (2009) 